Musées, Patrimoine et Culture scientifiques et techniques

$126 \mid 2009$

novembre - décembre 2009

\title{
Entretien avec René Rizzardo
}

Interview with René Rizzardo

\section{Florence Belaën}

URL : http://journals.openedition.org/ocim/208

DOI : 10.4000/ocim.208

ISSN : 2108-646X

Éditeur

OCIM

Édition imprimée

Date de publication : 1 novembre 2009

Pagination : 24-27

ISSN : 0994-1908

Référence électronique

Florence Belaën, «Entretien avec René Rizzardo », La Lettre de l'OCIM [En ligne], 126 | 2009, mis en ligne le 01 novembre 2011, consulté le 03 mai 2019. URL : http://journals.openedition.org/ocim/208 ; DOI : 10.4000/ocim.208 


\section{Entretien avec René Rizzardo*}

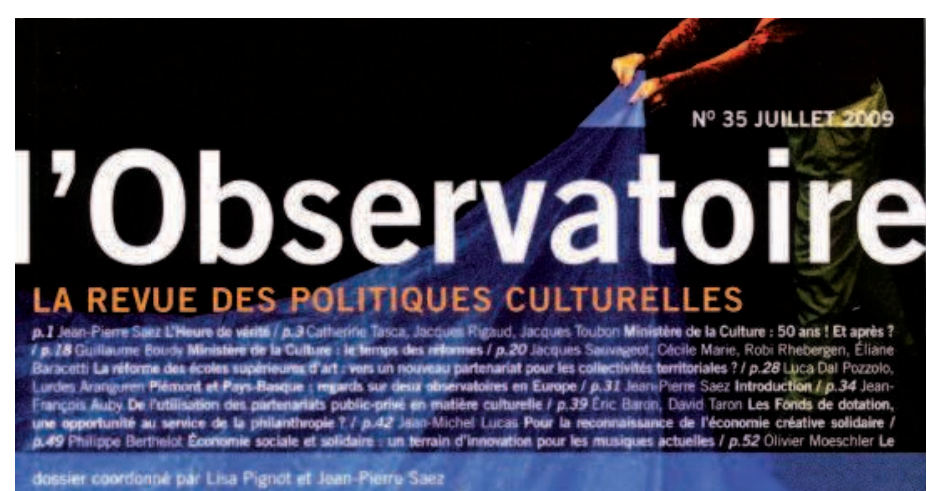

LES RAPPORTS PUBLIC/PRIVÉ DANS LA CULTURE

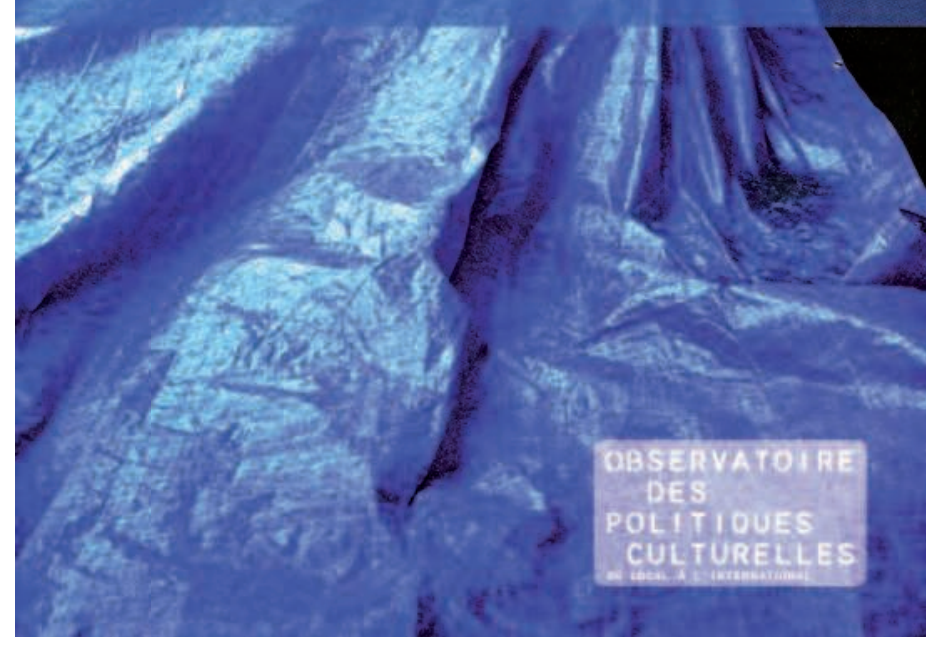

* René Rizzardo est directeur honoraire de l'Observatoire des Politiques Culturelles qu'il a dirigé de 1989 à 2002
Outil de connaissance et d'accompagnement au service des collectivités territoriales sur leurs politiques culturelles, l'Observatoire des Politiques Culturelles (OPC) est né en mars 1989 à Grenoble. Vingt ans après, René Rizzardo revient sur les circonstances qui ont présidé à sa création, en abordant notamment la question de l'évaluation dans les politiques publiques et son lien avec l'observation.

Pouvez-vous nous dire comment est né l'Observatoire des politiques culturelles de Grenoble?

L'Observatoire n'est pas né d'une commande, mais d'une démarche du Département des Études et de la Prospective (DEP). Ce service du ministère de la Culture a très tôt pris en compte l'émergence des politiques culturelles des collectivités territoriales, notamment à travers des études sur leurs dépenses culturelles. Il s'est également intéressé aux relations partenariales entre le ministère de la Culture et les collectivités. Avec le doublement du budget culturel en 1981 et le lancement des conventions de développement culturel, on assiste à une montée en puissance des collectivités favorisée par la décentralisation initiée par Gaston Defferre, dans un effet d'entraînement indéniable qui va conforter les collectivités les plus engagées, les villes surtout, et stimuler les nouvelles ambitions territoriales.

En 1986, Augustin Girard, chef du DEP fait réaliser une étude intitulée Douze villes et le changement 
culturel, qui porte sur les effets des conventions de développement culturel signées par ces villes tant sur leurs objectifs et leurs projets que sur ce qu'elles attendent du ministère de la Culture, la question étant : quelle est la part d'autonomie des collectivités dans la coopération et la décision partagée ? Cette étude fera l'objet d'une restitution auprès des villes, à Avignon où je suis alors chargé de mission pour le DEP, au Centre National de Formation d'Avignon.

Dans le même temps, le DEP lance en relation avec les directions régionales des affaires culturelles, un programme de six rencontres régionales sur la coopération entre les collectivités territoriales et le ministère, chaque séminaire en région étant précédé d'une étude sur les financements croisés, résultats concrets de cette coopération pour introduire la question : quel est l'apport de chaque partenaire public au financement des principales institutions artistiques et culturelles ou à des programmes décidés en commun, en fonctionnement comme en investissement, et quel bilan peut-on tirer des acquis et des limites de la coopération, quels effets pervers peut-elle avoir?

Ces séminaires régionaux très suivis ont d'une certaine manière installé un objectif - comprendre comment le développement culturel se construit dans la durée par le partenariat public et au profit de quels enjeux - mais également une méthode - à partir d'études préalables mettre en débat toutes les questions que pose le développement culturel, en quelque sorte une observation très concrète en grandeur nature avec les protagonistes directement impliqués.

Le bilan de ces rencontres sera présenté au colloque fondateur de l'Observatoire des politiques culturelles (OPC), à Grenoble en décembre 1988, puisque en effet, Augustin Girard va convaincre les services du ministère de la nécessité de pérenniser la démarche, mais en la déléguant à un organisme extérieur, dont il me demande de concevoir la forme et les partenaires.

Nous l'appellerons «Observatoire », terme un peu passe-partout, ne disposant d'ailleurs pas de fonction statistique, cette dernière étant au DEP, en complémentarité totale avec lui. Quant aux partenaires, nous les trouvons à Grenoble où l'Institut d'études politiques a une compétence très large en matière de recherche culturelle et dispose déjà du premier DESS de direction de projet culturel mis en oeuvre au Centre National de Formation d'Avignon, organisme qui sera supprimé en 1987, l'Observatoire installé début 1989, reprenant ce diplôme à son compte pour la formation continue des cadres culturels des collectivités territoriales. Un partenariat s'engage également avec l'université Pierre MendèsFrance très engagée sur les questions territoriales, la Région Rhône-Alpes qui apporte une part du financement de départ et la Ville de Grenoble qui met les locaux à disposition. Le conseil d'administration de l'association va donc réunir l'État, deux collectivités, les universités grenobloises, le Centre National de la Fonction Publique Territoriale (CNFPT), des personnalités qualifiées, élus cooptés à titre personnel et chercheurs d'autres régions, l'objectif étant d'assurer aux débats à venir pour orienter les travaux de l'Observatoire, l'assise partenariale à l'origine de sa fondation et l'appui de la recherche pour garantir la crédibilité et le caractère public de ses travaux.

\section{Comment l'Observatoire s'inscrit-il dans le paysage culturel et institutionnel au moment de sa création?}

De deux manières : d'une part comme un outil d'accompagnement des collectivités territoriales en matière culturelle ; d'autre part comme une interface entre les questions culturelles qui se posent dans les territoires, et les collectivités publiques.

Pour le premier positionnement, les études les rencontres, la publication de synthèses, les séminaires organisés en commun entre les directions régionales des affaires culturelles, les collectivités, parfois le CNFPT, pour élaborer des problématiques ou rendre compte de travaux, sont les modes opératoires privilégiés. La formation des cadres culturels territoriaux en cours d'emploi, est naturellement un des points fort de cet «accompagnement». Le second positionnement s'est traduit d'abord par la constitution d'un réseau de chercheurs spécialistes de questions spécifiques ou généralistes, l'Observatoire jouant à cet égard également la fonction d'interface entre la recherche et la demande publique. Cet enjeu s'est traduit par la mise en place d'un conseil scientifique durant les trois premières années, par la suite, la méthode de relation avec les chercheurs ayant évolué vers des séminaires réguliers.

La méthode peut se résumer ainsi : une question proposée par l'OPC, une démarche problématique décidée en commun, une équipe de chercheurs, un partenariat institutionnel et financier (l'Observatoire ne dispose pas de crédits pour ses travaux), la mise en oeuvre d'un programme, la restitution auprès des 
partenaires concernés, des publications. Rajoutons la création rapide d'un bulletin dont le rythme annuel s'est accéléré. Si les thématiques ont bien sûr évolué dans le temps, la méthode n'a guère varié, ayant fait ses preuves, les principes étant toujours d'actualité, en particulier le caractère public de ses travaux, principe parfaitement admis par les collectivités, protégeant l'Observatoire de toute demande d'audit.

\section{Quelle place a pris la question de l'évaluation dans vos missions?}

Elle est très vite apparue comme essentielle au regard des questions posées sur les objectifs mêmes des politiques culturelles, d'autant qu'avant même sa création, deux évaluations emblématiques, celles d'Annecy et de Rennes avaient été suivies de très près par le DEP.

Décider d'évaluer, c'est donner de la valeur et de l'importance à une activité, à une question, à un objectif, terme sans lequel il n'y a pas vraiment d'évaluation. Mais évaluer c'est aider à redéfinir l'objectif en fonction d'un contexte, de l'évolution des moyens associés à cet objectif, des résultats constatés. L'évaluation suppose une méthode qui peut être adaptée à la nature de ce que l'on évalue. Cette question de méthode nous a conduit à mettre très vite en chantier un séminaire qui a donné lieu à une publication commune avec le DEP, publiée à La Documentation française, afin de lancer le débat mais aussi proposer une méthodologie d'une évaluation non pas quantitative, mais qualitative : comprendre la complexité d'une situation et les raisons du succès ou de l'échec des objectifs affichés, afin de la transformer, y compris par la formation. L'évaluation est devenue un axe privilégié de travail et a fait récemment l'objet d'un des colloques du 20e anniversaire de l'Observatoire à Annecy, un retour aux sources.

\section{Quelle relation avez-vous établie entre observation et évaluation?}

Ces deux démarches se nourrissent mutuellement, encore faut-il qu'elles fassent l'objet de stratégies conjointes dans les territoires et au plan national, ce qui est rarement le cas. La production d'informations permanentes par l'observation, notamment sur le contexte dans lequel se déroule une action ou une politique, peut faciliter une démarche d'évaluation. Nous avons établi naturellement ce lien, les travaux généraux de l'Observatoire apportant des éléments de contexte et comparatifs. Nous avons veillé également à distinguer dans des situations précises, ce qui relevait d'études et ce qui relevait d'évaluations. L'OPC est crédité d'un rôle de conseil en la matière. Lorsqu'une collectivité formule une demande à l'Observatoire elle fait l'objet d'un débat sur les attentes, les résultats attendus, les intentions qui sous-tendent cette demande. L'étude peut répondre à une question commune à plusieurs collectivités, comme l'étude comparative sur les compétences culturelles de cinq agglomérations. L'évaluation va d'abord se référer à une situation pour laquelle on cherche à comprendre ce qui se passe, pourquoi ça marche ou non, où sont les limites dans la réalisation des objectifs, comment faire évoluer la situation.

On observe une demande d'observatoires régionaux sur des questions ciblées, comme l'emploi culturel ou l'économie du spectacle vivant. Reste à savoir si les données permanentes qui en résultent vont favoriser l'évaluation dans ces secteurs ou simplement éclairer la décision politique.

Le contexte change en matière d'évaluation des politiques publiques, alors que depuis le rapport de Patrick Viveret et la circulaire de Michel Rocard alors premier ministre en 1988, l'intérêt pour l'évaluation est resté faible. Mais son rôle est relancé pour de bonnes raisons (la complexité des problèmes à résoudre) et pour de moins bonnes motivations (l'obsession de la performance, affichée telle quelle dans la lettre du président de la République à la ministre de la Culture de l'époque). Le risque est en effet que l'évaluation soit détournée de sa finalité démocratique au profit du contrôle, ce qui n'est pas son rôle ${ }^{(1)}$.

Il est indispensable dans ce contexte d'être très précis sur l'usage de l'évaluation comme de la conception de l'observation, complémentarité ne devant pas être confondue avec soumission réciproque.

\section{Qu'est-ce qui à vos yeux, a fait défaut à l'Observatoire ou à côté de quoi est-il passé ?}

J'exprimerai deux regrets. Le premier concerne le caractère institutionnel dominant de l'Observatoire qui nous a freiné sur une intention que nous avons eu quelques années après la création. À la suite des projets culturels de quartiers lancés par la ministère nous avions pensé mettre en place une banque de données des expériences artistiques et culturelles innovantes. Pour diverses raisons, nous ne l'avons pas fait et je pense que cette démarche aurait élargi le champ de connaissance et d'analyse de 
l'Observatoire et abouti à une mise en réseau d'expériences de nature proche. Personne d'ailleurs ne l'a fait. Nous avions les outils, avec les chercheurs, pour qualifier l'innovation, question toujours délicate et surtout pour développer des évaluations. Cette base de données aurait pris son sens dans la démarche de l'Observatoire puisqu'il est en mesure de relier analyses de situations et apports d'expérimentations pour créer du mouvement.

Le ministère de la Culture n'a pas compris alors la nécessité de nourrir la notion même de politique culturelle par l'innovation, le risque étant si cette interactivité fait défaut, que l'on consacre l'énergie à gérer l'existant. Certes les travaux de l'Observatoire n'ignorent pas les expériences innovantes, mais c'est l'effet réseau qui aurait gagné avec la banque de données. Sans compter l'apport pour la formation.

En deuxième lieu, je pense que nous aurions dû plus rapidement mettre en place des programmes d'évaluation concertée sur un même sujet avec un réseau de territoires confrontés aux mêmes interrogations. Je pense notamment à l'éducation artistique. Mais lorsque nous avons proposé cette idée à l'Institut
National de Recherche Pédagogique, il a décliné et le ministère de la Culture n'a pas embrayé, plus préoccupé à annoncer de nouvelles initiatives que d'évaluer l'existant pour en tirer les enseignements. Cela se fait pour les études, mais l'évaluation peut apporter à un réseau de décideurs, d'acteurs, voire d'usagers, une autre dimension, celle formative qui consiste à s'approprier des situations pour les transformer.

Ces regrets sont provisoires, car l'Observatoire d'aujourd'hui est tout à fait en mesure d'aller dans ce sens sur les nouveaux thèmes pris en compte, comme la diversité culturelle ou le développement artistique dans les territoires. Quant à l'éducation artistique, elle redevient d'actualité.

Il y a en fait un temps pour tout et ce qui importe c'est d'être un « observatoire guetteur » de ce qui bouge pour sans cesse faire aussi bouger les lignes.

\section{Note}

(1) Voir l'article de Julien Damon « La fièvre de l'évaluation » dans la revue Sciences Humaines d'octobre 2009. 